\title{
Doença de Sutton: Diagnóstico, manejo e opções terapêuticas
}

\author{
Sutton's disease: Diagnosis, management and therapeutic options \\ Enfermedad de Sutton: Diagnóstico, manejo y opciones terapéuticas
}

Izabela Fornazari Delamura

ORCID: https://orcid.org/0000-0001-8046-489X Universidade Estadual Paulista, Brasil

E-mail: izabela.delamura@unesp.br

Murilo de Carvalho Souza

ORCID: https://orcid.org/0000-0002-8445-0724 Universidade Estadual Paulista, Brasil

E-mail: murilo.carvalho@unesp.br

Arthur Henrique Alécio Viotto

ORCID: https://orcid.org/0000-0002-5368-5336

Universidade Estadual Paulista, Brasil

E-mail: arthur.viotto@unesp.br

Ruan Henrique Delmonica Barra

ORCID: https://orcid.org/0000-0002-7929-1618

Universidade Estadual Paulista, Brasil

E-mail: ruan_d.barra@hotmail.com

Luis Fernando Alves da Silva

ORCID: https://orcid.org/0000-0002-5761-5554

Universidade Estadual Paulista, Brasil E-mail: luis.f.silva@unesp.br

Vinicius Ferreira Bizelli

ORCID: https://orcid.org/0000-0003-1813-3509 Universidade Estadual Paulista, Brasil E-mail: v.bizelli@unesp.br

Ana Maira Pereira Baggio

ORCID: https://orcid.org/0000-0002-9474-5091 Universidade Estadual Paulista, Brasil E-mail: anamairabaggio@gmail.com

Stéfani Caroline Ferriolli

ORCID: https://orcid.org/0000-0003-0019-6026 Universidade Estadual Paulista, Brasil E-mail: stefani.ferriolli@outlook.com

Daniela Ponzoni

ORCID: https://orcid.org/0000-0001-5928-0914 Universidade Estadual Paulista, Brasil

E-mail: daniela.ponzoni@unesp.br

Ana Paula Farnezi Bassi

ORCID: https://orcid.org/0000-0002-0031-4953 Universidade Estadual Paulista, Brasil E-mail: ana.bassi@unesp.br

\section{Resumo}

A estomatite aftosa recorrente (EAR) é uma alteração mais comum da mucosa oral e se caracteriza por uma lesão ulcerada, bem definida, dolorosa, com centro necrótico, bordas elevadas e halo eritematoso, levando a um impacto negativo na qualidade de vida dos pacientes. Entretanto, ainda não ocorreu a caracterização de um agente etiológico específico para essa afecção. A EAR possui três formas principais, sendo a EAR menor a mais comum na cavidade bucal, as quais são ulcerações bem definidas, dolorosas e cicatrizam em torno de 10 a 14 dias. A herpetiforme, que possui várias lesões e podem coalescer entre 7 e 10 dias, por fim temos a EAR maior, a qual pode perdurar por mais de 6 semanas e podem deixar cicatrizes. O diagnóstico das lesões é feito após um exame clínico minucioso. Metodologia: Este estudo é um relato de caso de forma qualitativa e descritiva, trazendo dados relevantes da literatura. o ético os princípios da Declaração de Helsinque foram respeitados e o paciente assinou o "Termo de Consentimento Livre e Esclarecido" para participar da pesquisa. O objetivo deste estudo foi relatar um caso de EAR em um paciente do gênero masculino de 41 anos de idade com doença de Sutton, apresentando múltiplas úlceras necrosantes dentro de 9 anos de recidivas. Conclusão: Por ser a etiologia da EAR ainda desconhecida e uma desordem comum e recorrente na cavidade bucal o tratamento atual é 
diversificado e atenta-se no alívio dos sintomas da doença, variando, desde o uso de produtos naturais, uso tópico, antiinflamatórios, imunomoduladores e aplicação do laser de baixa potência.

Palavras-chave: Estomatite aftosa; Tratamento; Patologia; Úlceras orais.

\begin{abstract}
Recurrent aphthous stomatitis (RAS) is a more common disorder of the oral mucosa and is characterized by a well-defined, painful ulcerated lesion with a necrotic center, raised borders, and an erythematous halo. However, the characterization of a specific etiological agent for this condition has not yet occurred. RAS has three main forms, with the smallest RAS being the most common in the oral cavity, which are painful and well-defined ulcerations that heal within 10 to 14 days. Herpetiformis, which has several lesions and can coalesce between 7 and 10 days, finally we have the longest RAS, which can last for more than 6 weeks and can leave scars. Methodology: This study is a qualitative and descriptive case report, bringing relevant data from the literature. The ethical principles of the Declaration of Helsinki were respected and the patient signed the "Informed Consent Form" to participate in the research. The aim of this study was to report a case of RAS in a 41-year-old male patient with Sutton's disease, presenting multiple necrotizing ulcers within 9 years of recurrence. Conclusion: As the etiology of RAS is still unknown and a common and recurrent disorder in the oral cavity, current treatment is diversified and focuses on relieving the symptoms of the disease, ranging from the use of natural products, topical use, anti-inflammatory drugs, immunomodulators and low power laser application.
\end{abstract}

Keywords: Aphthous stomatitis; Treatment; Pathology; Oral ulcers.

\title{
Resumen
}

La estomatitis aftosa recurrente (EAR) es un trastorno más común de la mucosa oral y se caracteriza por una lesión ulcerada dolorosa y bien definida con un centro necrótico, bordes elevados y un halo eritematoso. Sin embargo, aún no se ha realizado la caracterización de un agente etiológico específico para esta afección. El RAS tiene tres formas principales, siendo el RAS más pequeño el más común en la cavidad oral, que son ulceraciones dolorosas y bien definidas que cicatrizan en 10 a 14 días. Herpetiformis, que tiene varias lesiones y puede coalescer entre 7 y 10 días, finalmente tenemos el RAS más largo, que puede durar más de 6 semanas y puede dejar cicatrices. Metodología: Este estudio es un reporte de caso cualitativo y descriptivo, aportando datos relevantes de la literatura. Se respetaron los principios éticos de la Declaración de Helsinki y el paciente firmó el "Formulario de consentimiento informado" para participar en la investigación. El objetivo de este estudio fue reportar un caso de EAR en un paciente masculino de 41 años con enfermedad de Sutton, que presenta múltiples úlceras necrotizantes dentro de los 9 años de recidiva. Conclusión: Como la etiología del EAR aún se desconoce y es un trastorno común y recurrente en la cavidad oral, el tratamiento actual está diversificado y se enfoca en aliviar los síntomas de la enfermedad, que van desde el uso de productos naturales, uso tópico, medicamentos antiinflamatorios, inmunomoduladores y aplicación de láser de baja potencia.

Palabras clave: Estomatitis aftosa; Tratamiento; Patología; Úlceras orales.

\section{Introdução}

A estomatite aftosa recorrente é uma das doenças mais comuns da mucosa oral. O termo "aftoso" é derivado de uma palavra grega "aphtha” que significa ulceração (Jonathan et al., 1996). A afta é um aspecto semiológico particular da ulceração mucosa, dolorosa, inflamatória e recorrente (Loïcet al., 2016). A prevalência relatada na população em geral varia de 5\% a $66 \%$, com uma média de 20\%. Diversos subgrupos de pacientes parecem apresentar diferentes causas para a ocorrência das aftas. As úlceras aftosas afetam até $25 \%$ da população em geral e as taxas de recorrência em 3 meses chegam a 50\% sendo mais comum em mulheres (Bassel et al., 2015).

Sua etiologia é multifatorial, estando associadas causas de origem local, como os traumatismos, ou sistêmicas, como as infecções e as doenças imuno-hematológicas (Paula et al., 2002). Os locais mais frequentes da alteração são a mucosa labial (30\%), a língua $(26 \%)$ e a mucosa jugal (22\%) (Heron et al., 2013).

Uma anamnese detalhada do paciente é crítica na avaliação de lesões ulcerativas orais e deve incluir a história médica completa, investigar traumas prévios na região, há quanto tempo a lesão está presente, a frequência dos episódios em casos recorrentes, a presença ou ausência de dor e o crescimento da lesão ao longo do tempo (Sarah et al., 2019). Devido à ausência de exames bioquímicos e histopatológicos específicos, o diagnóstico normalmente se baseia na história do paciente e na apresentação clínica de suas lesões (José Alexandre et al., 2008). 
As aftas são causadas por vasculite leucocitoclástica. Isso induz a necrose do tecido na origem de uma ulceração primária, cujo diâmetro e profundidade variam de acordo com o tamanho do vaso afetado (Loïcet al., 2016). Foram descritas três formas clínicas de úlceras aftosas: úlceras aftosas menores, úlceras aftosas herpetiformes e úlceras aftosas maiores. Muitas outras condições que se apresentam com lesões orais bolhosas ou vesiculosas ou ulcerações e erosões podem ser confundidas com úlceras aftosas (Loïcet al., 2016).

As ulcerações aftosas menores (aftas de Mikulicz) são as mais comuns e representam o padrão presente em mais de 80\% dos indivíduos afetados. As ulcerações aftosas maiores (doença de Sutton ou periadenite mucosa necrótica recorrente (PMNR) ocorrem em cerca de $10 \%$ dos pacientes encaminhados para tratamento e o restante apresentam ulcerações aftosas herpetiformes (Brad et al., 2011).

A forma maior, é de ocorrência rara, porém, de curso mais severo, sendo conhecida como úlceras de Sutton ou periadenite recorrente necrótica da mucosa (Paula et al., 2002) e afeta cerca de 10-15\% dos pacientes. As úlceras excedem $1 \mathrm{~cm}$ de diâmetro (Jonathan et al., 1996), acometendo mais frequentemente lábios e palato mole, mucosa mastigatória, dorso da língua ou gengiva que pode ser ocasionalmente envolvida, mas, podem aparecer em qualquer região da mucosa oral (Jonathan et al., 1996; Paula et al., 2002). Se manifestam geralmente após a puberdade de forma crônica, persistindo por 20 anos ou mais. O curso é imprevisível e pode incluir longos períodos de remissão ou intensa atividade, podendo se alternar em um ritmo aleatório, deixando cicatrizes (José Alexandre et al., 2008).

Outra doença importante a ser citada é a síndrome de Behçet. As manifestações clínicas são atribuídas à presença de vasculite, resultando em dano vascular, que se manifesta em um alto percentual de úlceras e lesões cutâneas, lesões inflamatórias dos olhos, sendo que as aftas correspondem a uma das características principais, podendo ser a manifestação mais precoce da doença (Elen et al., 2018; María Elena et al., 2016). Sua etiologia permanece desconhecida, porém a hipótese mais aceitável da doença é a de que hospedeiros geneticamente susceptíveis desencadeiam uma resposta inflamatória causada por um agente infeccioso. $\mathrm{O}$ fator de risco genético mais fortemente associado à síndrome de Behçet é o antígeno leucocitário humano (HLA)B51, que ocorre em cerca de 60\% dos pacientes com a doença (Elen et al., 2018).

O acompanhamento com o cirurgião dentista é essencial para controle do biofilme oral, manejo das ulcerações que venham a ocorrer e para prevenção e tratamento de infecções oportunistas que podem aparecer em períodos de imunossupressão (Larissa et al., 2019). O alívio das manifestações bucais pode ser feito com preparações tópicas de anestésicos locais (lidocaína 2\%) em aftas menores ou preparação local de esteróides que aderem à mucosa oral, como a pasta oral de triancinolona acetonida, que, aplicada localmente várias vezes ao dia, proporciona alívio dos sintomas e pode diminuir a duração da ulceração.

Como a doença afeta vários sistemas, os pacientes podem necessitar de cuidados de várias especialidades médicas, por isso é aconselhável o acompanhamento de um médico de atenção primária para coordenar o tratamento e o monitoramento (Elen et al., 2018; María Elena et al., 2016).

\section{Metodologia}

Este estudo trata-se de um relato de caso de um paciente jovem, sexo masculino, 30 anos de idade que procurou atendimento da Unesp - Araçatuba. O manejo clínico foi descrito de maneira qualitativa e descritiva (Pereira et al., 2018), trazendo dados relevantes da literatura a respeito de diagnóstico, manejo e opções terapêuticas da doença de Sutton. O estudo teve aprovação do Comitê em Pesquisa assim como seguiu os princípios éticos da Declaração de Helsinque foram respeitados e o paciente assinou o "Termo de Consentimento Livre e Esclarecido" para participar da pesquisa. 


\section{Caso Clínico}

Paciente de gênero masculino, branco, 30 anos, procurou atendimento no ambulatório de cirurgia da Faculdade de Odontologia de Araçatuba - Unesp em 2003, em virtude de três lesões ulceradas em língua (Figura 1), dolorosas, com evolução de quatro dias. Durante a anamnese relatou que lesões do mesmo aspecto, tamanho e localização vinham surgindo e regredindo há cinco anos. Anteriormente esteve sob acompanhamento no Hospital das Clínicas da Universidade de São Paulo por dois anos, onde foi submetido a exames diagnósticos para sífilis e HIV, além de videoendoscopia do aparelho digestivo. Nenhuma doença foi diagnosticada. A história médica e familiar não revelou doenças de base, nenhuma medicação em uso, histórico de alergias, doenças auto-imunes ou neoplásica.

Figura 1: Aspecto inicial da lesão durante o exame intraoral do lado direito, em 2003.

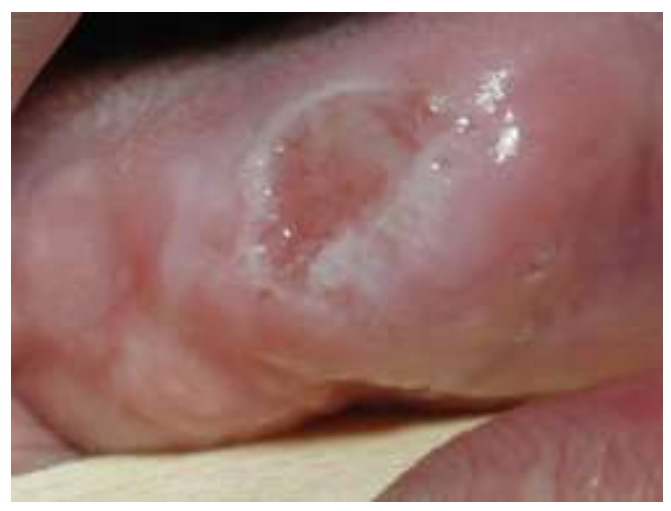

Fonte: Autores.

No exame físico foram observadas três úlceras na língua: uma em bordo lateral direito, com 3,5 centímetros em seu maior diâmetro, outra em bordo lateral esquerdo, com aproximadamente 1,5 centímetros de diâmetro e uma terceira em região de ápice lingual, com 2,0 centímetros de diâmetro. As lesões apresentavam aspecto clínico semelhante, bordas nítidas e escleróticas e discretamente elevadas, leito fibrinoso, alternado com algumas regiões eritematosas. A lesão foi biopsiada e o paciente tratado sintomaticamente com complexo B associado a vitamina $\mathrm{C}$, tratamento tópico com pomadas anestésicas e Omcilon orabase para melhorar a condição alimentar. A lesão regrediu em aproximadamente 40 dias e o exame histopatológico revelou quadro compatível com Estomatite Aftosa Recorrente.

Passados três anos (2005), o paciente retorna com lesões semelhantes, de maior extensão, novamente em bordos laterais (Figura 2: A e B) e ápice de língua. Foi encaminhado para avaliação pelo infectologista, que descartou possibilidade de lesão infecciosa. Foi instituído tratamento com corticosteróides por via oral com $20 \mathrm{mg}$ de prednisona ao dia, em doses decrescentes, durante 10 dias. Ao final do tratamento observou-se melhora do quadro clínico, que evoluiu para a completa resolução. Mais três recidivas foram observadas ao longo daquele ano e do ano seguinte, durante as quais, tratamentos com corticóides sistêmicos, tópicos, vitaminas do complexo B e C, analgésicos narcóticos (cloridrato de tramadol) e anestésicos tópicos foram utilizados, sempre observando uma regressão lenta e gradual ao longo de quatro a oito semanas, no entanto com recidivas constantes. 
Research, Society and Development, v. 10, n. 10, e06101018056, 2021

(CC BY 4.0) | ISSN 2525-3409 | DOI: http://dx.doi.org/10.33448/rsd-v10i10.18056

Figura 2: A: aspecto da lesão em rebordo de língua, lado esquerdo em 2005.

B: aspecto da lesão em rebordo de língua, lado direito em 2005.

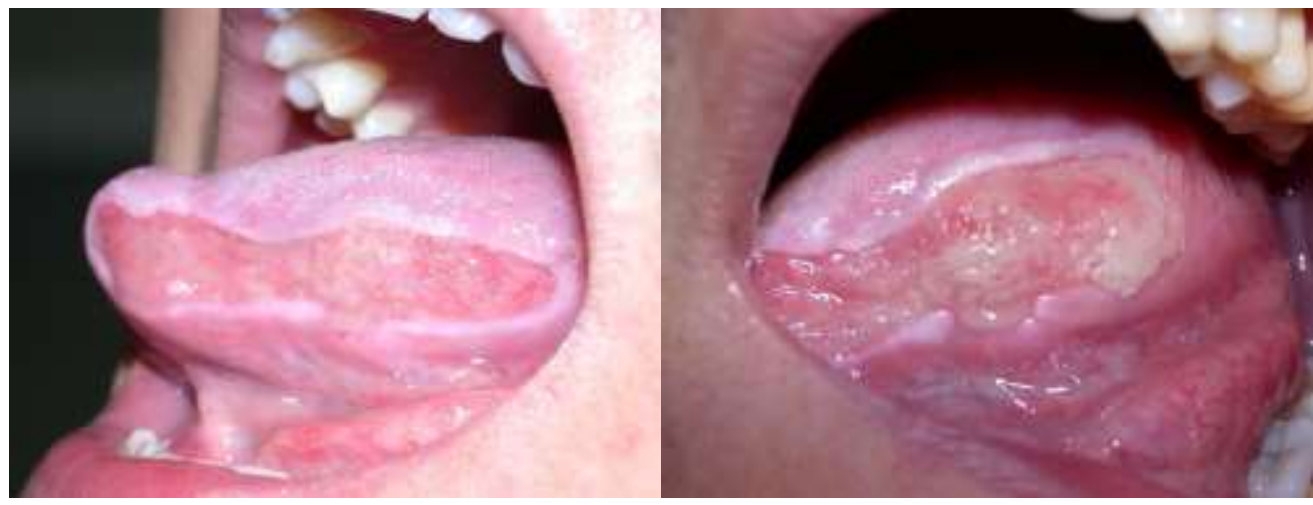

Fonte: Autores.

Em dezembro de 2006, paciente retorna com novas lesões no rebordo de língua (Figura 3: A e B) e ápice de língua (Figura 4).

Figura 3: A: aspecto da lesão em rebordo de língua, lado direito em dezembro de 2006.

B: aspecto da lesão em rebordo de língua, lado direito em dezembro de 2006.

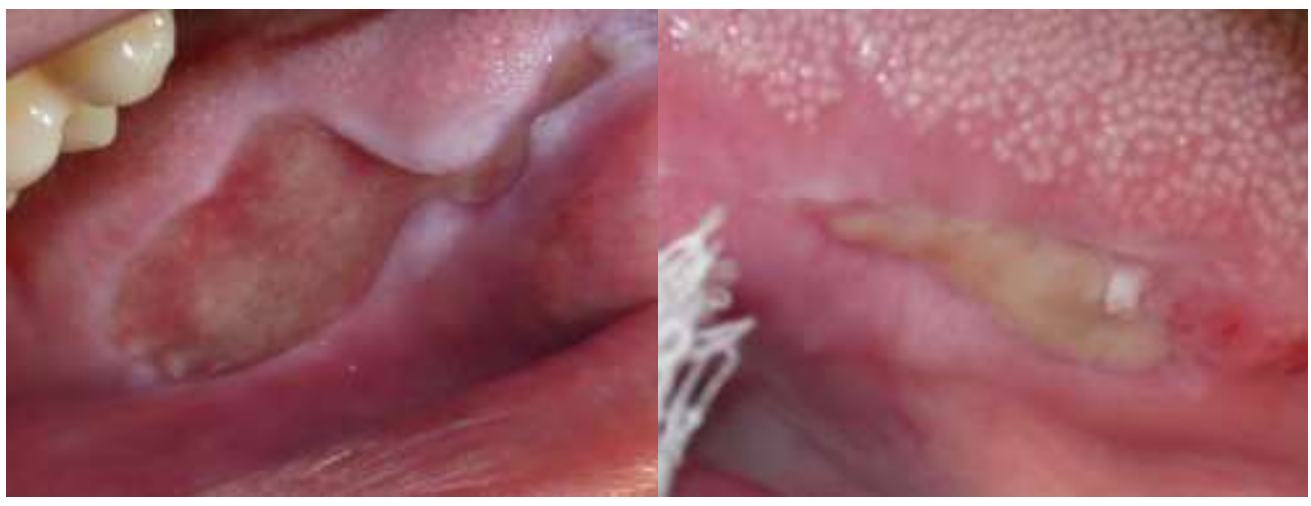

Fonte: Autores.

Figura 4: aspecto inicial da lesão durante o exame intra oral em ápice de língua.

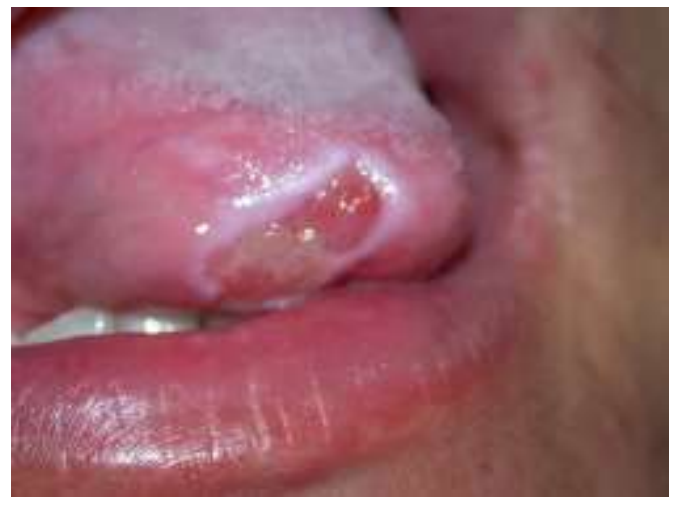

Fonte: Autores. 
Uma segunda biópsia incisional foi realizada em lesões localizadas em rebordo de língua nas regiões leucoplásicas em março de 2007 (figura 6: A e B) e o material foi encaminhado para exame anátomo-patológico, cujo resultado foi Afta de Sutton. Os cortes histológicos revelaram mucosa bucal ora revestida por epitélio pavimentoso estratificado paraqueratinizado, ora ulcerado (figura 7). No tecido conjuntivo observa-se áreas de necrose e intensa vasculite, onde é possível observar vasos ingurgitados por leucócitos e leve destruição da parede vascular, por degranulação dos mesmos. Presença de filetes nervosos em meio às fibras colágenas e numa faixa mais superficial, nas áreas sem epitélio, um intenso infiltrado inflamatório crônico, constituído principalmente por macrófagos e linfócitos, mais superficialmente, observa-se eventuais neutrófilos.

$\mathrm{O}$ tratamento com corticóides sistêmicos com betametasona foi novamente iniciado, sem resultados clínicos aparentes. O laser de baixa potência também foi utilizado sobre as lesões, que regrediram após dois meses. Três novas recidivas surgiram até 2009, onde novamente instituiu-se terapia com corticóides tópicos e sistêmicos.

Figura 6: A: aspecto cirúrgico antes da realização da biópsia.

B: aspecto cirúrgico após a biópsia incisional.

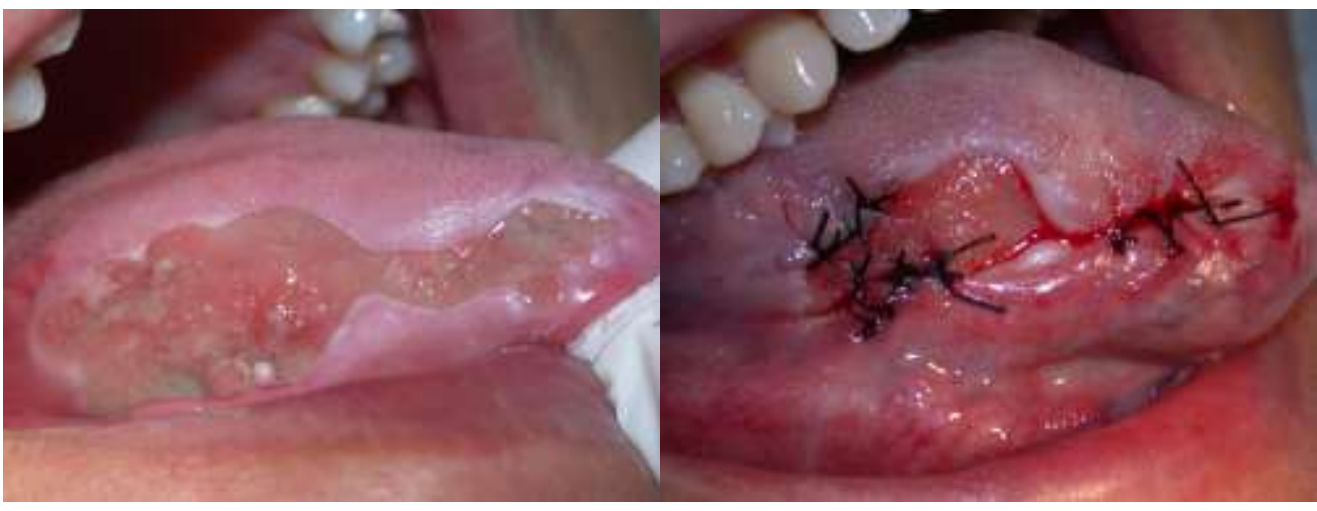

Fonte: Autores.

Figura 7: Aspecto histopatológico da lesão

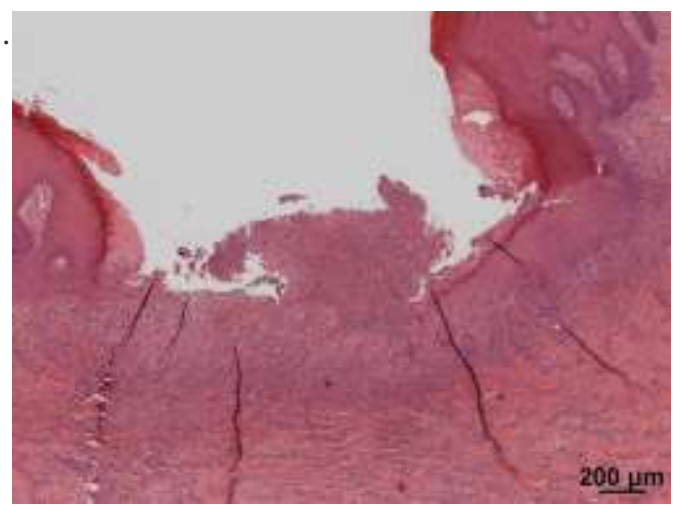

Fonte: Autores.

\section{Fatores predisponentes}

O mecanismo patogenético das aftas é multifatorial. Os dados atualmente conhecidos sugerem uma doença auto-imune e a maior frequência de autoanticorpos antitireoidianos (29\% vs. 18\% em indivíduos controle) reforça essa hipótese (Loïcet al., 
2016). Além disso, indivíduos com história familiar de aftas, não fumantes e com diminuição da vitamina B12, vitamina $C$ e folato estão em maior risco de desenvolver aftas (Sarah et al., 2019).

Uma predisposição genética foi sugerida porque certos antígenos de histocompatibilidade, como HLA-B12, B51 e Cw7, foram associados a grupos de pacientes que sofrem de aftas e, estudos de famílias com gêmeos, confirmaram a existência de uma predisposição genética (Loïcet al., 2016). 2515

$\mathrm{O}$ trauma podendo ser físico e/ou químico, tem sido relatado por pacientes, clínicos e estudiosos como fator desencadeante no desenvolvimento das lesões da EAR. Os pacientes referem como causas mais comuns do traumatismo: escovação dentária, fio dental, goma de mascar, alimentos com espinha, má oclusão dentária, injeções e tratamentos dentários (Ana Raquel Monteiro Dia., 2013; Loïcet al., 2016; Sarah et al., 2019).

A deficiência nutricional pode também acarretar aftas. A ausência de elementos como ferro, folato, zinco e vitaminas B1, B2, B6 e B12, podendo estes estarem também associados a síndromes de mal absorção ou sensibilidade ao glúten (Ana Raquel Monteiro Dia., 2013).

Outro fator que pode ser destacado é o estresse, podendo resultar em episódios de estomatite aftosa recorrente não muito bem compreendido (Laura et al., 2012). Muitos estudos descobriram que estresse, depressão e ansiedade são fatores comumente observados em pacientes com aftas $\mathrm{O}$ estresse emocional frequentemente leva à exacerbação do caso clínico (Loïcet al., 2016; Kuan-Chou et al., 2019). Tem sido sugerido que níveis elevados de cortisol salivar ou de espécies reativas de oxigênio na saliva podem levar ao aparecimento de lesões (Laura et al., 2012). Devido ao estresse, os pacientes podem começar a mastigar os lábios ou realizar outros hábitos de enfrentamento que causem traumas na área (Laura et al., 2012). O aumento na frequência de aftas na população estudantil durante o período de exames é frequentemente dado como um exemplo de uma ligação entre estresse e aftas (Loïcet al., 2016).

Estressores mentais e físicos foram significativamente associados ao desenvolvimento de episódios de estomatite aftosa recorrente, no entanto os estressores mentais tiveram um efeito muito mais pronunciado. Entre as doenças da mucosa oral, descobriu-se que as EAR apresentam a maior proporção de pacientes com problemas psicológicos (José Alexandre et al., 2008).

Apesar da controvérsia sobre o papel direto do estresse na etiologia da EAR, parece evidente a associação dessas lesões com alterações do sistema imunológico, e também a relação dessas alterações imunológicas com o mecanismo de estresse. Essa desregulação na imunidade é endossada pela psiconeuroendocrinologia, que evidencia alterações em praticamente todos os níveis da resposta imunológica (José Alexandre et al., 2008).

Outros fatores menos comuns podem ser vistos como: fatores alérgicos/hipersensibilidade a certos alimentos como a chocolate, amendoim e cereais; fatores microbianos; vírus herpes humano 8, citomegalovírus, vírus Epstein-barr, vírus papiloma humano e vírus herpes simplex 1 e fatores iatrogênicos (Ana Raquel Monteiro Dia., 2013).

Uma minoria de pacientes com EAR tem ulceração oral cíclica relatada na fase luteínica do ciclo menstrual e, em vez disso, uma diminuição nos primeiros meses de gravidez e exacerbação no puerpério (José Alexandre et al., 2008; Paula et al., 2002) No entanto, não há ainda uma hipótese explicativa para esta associação (Ana Raquel Monteiro Dia., 2013).

\section{Discussão}

Histologicamente, a doença de Sutton é caracterizada por lesões inflamatórias do epitélio, tecido conjuntivo e até glândulas salivares acessórias, o infiltrado inflamatório onde os neutrófilos predominam, afetam vasos de maiores dimensões e calibre (Kamsu Huguette et al., 2020). O exato papel dos neutrófilos na patogênese da EAR ainda não está completamente esclarecidos, as funções de quimiotaxia e fagocítose, bem como a produção de radicais tóxicos de oxigênio, não se apresentaram exacerbadas 
em relação aos pacientes saudáveis, porém observou-se uma grande concentração dessas células na fase ulcerativa da lesão, sugerindo uma participação ativa (Elen et al., 2018).

Presume-se que os macrófagos desempenhem uma função de "limpeza" dos neutrófilos remanescentes na área de úlcera, pois, em estudo histopatológico, alguns autores observaram a presença de numerosos macrófagos carregados com fagolisossomos contendo restos de neutrófilos granulócitos (Loïcet al., 2016; Kamsu Huguette et al., 2020).

As aftas de Sutton, dificultam a execução das funções básicas dos pacientes como falar, mastigar, engolir e reduzem significativamente sua qualidade de vida. Elas podem persistir por várias semanas (entre 2 a 12 semanas), desenvolvendo endurecimento de suas bordas e deixando cicatrizes fibrosas, retráteis e mutilantes durante a cicatrização. O curso é imprevisível: longos períodos de remissão e intensa atividade podem se alternar em um ritmo aleatório (Loïcet al., 2016).

Embora nenhum agente desencadeante seja responsável, a destruição da mucosa parece representar uma reação imunológica mediada por células T, com produção do fator de necrose tumoral alfa (TNF-alfa). Esse fator é uma importante citocina inflamatória e auxilia na sinalização do epitélio de superfície para a destruição das células T citotóxicas (CD8+). São fortes as evidências de destruição da mucosa oral mediada por estes linfócitos, mas as causas iniciais são evasivas e, mais provavelmente, altamente variáveis (Brad et al., 2011).

Exames histopatológicos das lesões orais revelam infiltrado de células mononucleares nas lesões da fase pré-ulcerativa. Nas formas ulceradas há acúmulo de leucócitos polimorfonucleares e mastócitos foram encontrados em maior número nas lesões precoces de EAR. Estão reduzidos nos estágios mais avançados provavelmente pela degranulação ou destruição dos mastócitos. (Paula et al., 2002).

Devido ao fato da etiologia ainda ser desconhecida, não há um tratamento estabelecido para a cura das lesões até o momento (Bassel et al., 2015). A severidade das lesões (número, localização, tamanho e duração) e seus efeitos no paciente (grau de desconforto, efeito durante a alimentação e qualidade de vida) devem ser avaliados e, desta maneira, as formas de tratamento são voltadas para o alívio dos sintomas e cicatrização das úlceras (Gilliene et al., 2013; Paula et al., 2002). Além da terapêutica medicamentosa, como o fator emocional/estresse é um dos fatores desencadeantes da EAR, é indicado o tratamento em conjunto com um um profissional da área comportamental (psiquiatra ou psicólogo) (Daniela et al., 2005).

A utilização desses diferentes recursos terapêuticos se dá tanto de forma sistêmica, quanto de forma tópica. No entanto, a forma tópica de tratamento se mostra mais eficiente e segura para o paciente, com a de menor quantidade de efeitos colaterais, consistindo na primeira opção de tratamento para as aftas recorrentes (Gilliene et al., 2013).

Os medicamentos usados reduzem a dor e a freqüência de aparecimento das lesões. Regimes tópicos e sistêmicos de tetraciclinas têm sido utilizados no manejo da EAR, não somente devido às suas propriedades antimicrobianas, mas também devido a outras propriedades, tais como inibição da produção de prostaglandinas, supressão de leucócitos, inibição de colagenases e gelatinases (Anna Clara et al., 2015). Substâncias anti-inflamatórias esteroidais e não esteroidais, como a pomada de dexametasona (uso tópico) a pasta de amlexanox (uso tópico) e a combinação de levamisol e prednisolona (uso sistêmico) também podem ser utilizadas no tratamento da estomatite aftosa recorrente (Anna Clara et al., 2015). De modo geral o uso dos medicamentos sistêmicos é necessário visto a eficácia limitada dos agentes tópicos, quando a dor é intensa e/ou quando a permanência da úlcera é longa. Outros fármacos como corticosteróides, levamisole, colchicina, pentoxifilina, dapsona e talidomida também podem ser utilizados (Paula et al., 2002). No caso de inefetividade do suporte doméstico, ou a necessidade de administração endovenosa de esteróides ou outros fármacos, a internação hospitalar pode se fazer necessária (Daniela et al., 2005).

A laserterapia tem sido usada em diversas áreas da Odontologia por se tratar de uma ferramenta tecnológica que proporciona maior conforto aos pacientes e confiabilidade ao profissional cirurgião-dentista (Luiza Zanette et al. 2017) e, para o 
tratamento da EAR, o laser de CO2 em baixa potência, diodo de $940 \mathrm{~nm}$, láser $\mathrm{Nd}$ : YAG, podem ser utilizados como terapia auxiliar. Alguns desses lasers, a partir da sua primeira aplicação, já demonstraram resultados satisfatórios na sintomatologia dolorosa e maior rapidez na cicatrização (Shariq et al., 2016). A terapia fotônica, que é muito utilizada em pacientes oncológicos, também é uma possibilidade. A utilização do laser vermelho e infravermelho são indicados como agente antimicrobiano e analgésico, além de acelerarem o processo de reparo e cicatrização de lesões na mucosa (Luiza Zanette et al., 2017).

Para que ocorra um diagnóstico correto da lesão é indicado a realização de exames complementares, como, o anatomopatológico no qual biópsia incisional está indicada em todas as úlceras que persistem durante 3 ou mais semanas, associadas ou não a dor, solitárias ou múltiplas, lesões de origem indeterminada que persistem sem sinais de cura após 2 semanas ou aquelas que, identificada uma causa possível, não regridem após 2 semanas de tratamento adequado ou da remoção do fator desencadeante. A realização da biópsia é preconizada para excluir possíveis doenças malignas do diagnóstico final, uma vez que o resultado do exame para EAR não é específico da doença (Ana Raquel Monteiro Dia., 2013).

\section{Conclusão}

As aftas de Sutton, por serem uma variação de maior tamanho da Estomatite Aftosa Recorrente, assim como esta, tem seu diagnóstico obtido clinicamente e, devem ser biopsiadas para se descartar outras patologias, sendo que, os achados histopatológicos geralmente mostram ulceração inespecífica.

Apesar de ter havido progresso em relação à descrição, causas e tratamento da EAR, sua etiologia permanece obscura, tendo como principais fatores predisponentes os traumas físicos, deficiências nutricionais, fatores genéticos e estresse mental. Esse último merece destaque, pois, muitos estudos mostram uma incidência muito grande dos fatores de estresse, depressão e ansiedade em pacientes com EAR, sendo considerados, inclusive, fatores exacerbadores desse quadro.

Como terapia, tem-se como primeira linha os corticosteróides tópicos, embora casos complexos ou graves possam exigir esteróides sistêmicos ou medicamentos imunossupressores alternativos.

Visto que a sintomatologia dolorosa é um agravante a ser observado nas aftas de Sutton, estudos futuros que envolvam laserterapia e fármacos analgésicos e anti-inflamatórios seriam de grande relevância clínica em busca do bem estar do paciente.

A condução do caso clínico é muitas vezes desafiadora devido à possibilidade de ocorrer recidivas e ausência de tratamento definitivo.

\section{Referências}

Alfonso Valdés, M. E. (2016). Síndrome de Behcet. Revista Cubana de Hematología, Inmunología y Hemoterapia, $32(3), 301-315$.

Costa, G. B., \& Castro, J. F. (2013). Etiologia e tratamento da estomatite aftosa recorrente-revisão de literatura. Medicina (Ribeirão Preto), $46(1), 1-7$.

da Rocha Curvelo, J. A., de Carvalho Ferreira, D., de Carvalho, F. C. R., \& Janini, M. E. R. (2008). Úlceras aftosas recorrentes e sua possível associação ao estresse. Revista de Ciências Médicas e Biológicas, 7(1), 67-75.

de Araújo, A. R. M. D. (2013). Abordagem diagnóstica da Aftose oral recorrente.

de Souza Tolentino, E., Romanichen, I. M. M., Bessani, A., Nicácio, L. A., \& Ferreira, G. Z. (2018). Manifestações bucais e considerações gerais da síndrome de Behçet: relato de caso. Revista da Faculdade de Odontologia-UPF, 23(3), 322-328.

Fávaro, D. M., \& Martins, G. (2005). Ulceração aftosa recorrente em crianças: revisão. Archives of Oral Research, 1(3).

Fitzpatrick, S. G., Cohen, D. M., \& Clark, A. N. (2019). Ulcerated lesions of the oral mucosa: clinical and histologic review. Head and neck pathology, 13(1), 91102.

Fraiha, P. M., Bittencourt, P. G., \& Celestino, L. R. (2002). Estomatite aftosa recorrente: revisão bibliográfica. Revista Brasileira de Otorrinolaringologia, 68(4), 571-578.

Gonzaga, H. F. D. S., Gonzaga, L. H. D. S., Costa, C. A. D. S., Spolidorio, L. C., Osaki, L., \& Naka, P. M. (2013). Avaliação sistêmica de paciente portador de ulceração aftosa recorrente. Revista de Odontologia da UNESP, 24(2), 253-262. 
Research, Society and Development, v. 10, n. 10, e06101018056, 2021 (CC BY 4.0) | ISSN 2525-3409 | DOI: http://dx.doi.org/10.33448/rsd-v10i10.18056

Gürkan, A., Özlü, S. G., Altıylık-Özer, P., Kurtul, B. E., Karacan, C. D., \& Şenel, S. (2015). Recurrent aphthous stomatitis in childhood and adolescence: a singlecenter experience. Pediatric dermatology, 32(4), 476-480.

Huling, L. B., Baccaglini, L., Choquette, L., Feinn, R. S., \& Lalla, R. V. (2012). Effect of stressful life events on the onset and duration of recurrent aphthous stomatitis. Journal of oral pathology \& medicine, 41(2), 149-152.

Lin, K. C., Tsai, L. L., Ko, E. C., Yuan, K. S. P., \& Wu, S. Y. (2019). Comorbidity profiles among patients with recurrent aphthous stomatitis: A case-control study. Journal of the Formosan Medical Association, 118(3), 664-670.

Najeeb, S., Khurshid, Z., Zohaib, S., Najeeb, B., Qasim, S. B., \& Zafar, M. S. (2016). Management of recurrent aphthous ulcers using low-level lasers: a systematic review. Medicina, 52(5), 263-268.

Neville, B. (2011). Patologia oral e maxilofacial (12a ed.). Elsevier Brasil.

Pereira, A. S., Shitsuka, D. M., Parreira, F. J., \& Shitsuka, R. (2018). Metodologia da pesquisa científica.[e-book]. Santa Maria. Ed (pp. 3-9). UAB/NTE/UFSM. Disponível em: https://repositorio. ufsm. br/bitstream/handle/1/15824/Lic_Computacao_Metodologia-Pesquisa-Cientifica. pdf.

Pereira, K. M. A., Rocha, D. A. P., Galvão, H. C., \& Freitas, R. D. A. (2013). Ulceração aftosa recorrente: revisão dos conceitos atuais. Revista de Odontologia da UNESP, 35(1), 61-67.

Reolon, L. Z., Rigo, L., Conto, F. D., \& Cé, L. C. (2017). Impacto da laserterapia na qualidade de vida de pacientes oncológicos portadores de mucosite oral. Revista de Odontologia da UNESP, 46(1), 19-27.

Rioboo Crespo, M., \& Bascones Martínez, A. (2011). Aftas de la mucosa oral. Avances en Odontoestomatología, 27(2), 63-74

Ship, J. A. (1996). Recurrent aphthous stomatitis: an update. Oral Surgery, Oral Medicine, Oral Pathology, Oral Radiology, and Endodontology, 81(2), 141-147.

Silva, L. F., de Carvalho, L. F. L., de Souza Gorjão, P., dos Santos, A. P. P., da Silva, V. E. S., \& Fernandes, V. M. P. (2019). O papel do cirurgião-dentista no diagnóstico e tratamento de lesões orais associadas a doenças sistêmicas inflamatórias. Revista Interdisciplinar, 12(2), 121-125.

Sonia, K. H., \& Catherine, E. (2020). Sutton's Disease and Vitamin Deficiency: Diagnostic and Therapeutic Difficulties. About a Clinical Case at the University Teaching Hospital Center of Yaounde.

Tarakji, B., Gazal, G., Al-Maweri, S. A., Azzeghaiby, S. N., \& Alaizari, N. (2015). Guideline for the diagnosis and treatment of recurrent aphthous stomatitis for dental practitioners. Journal of international oral health: JIOH, 7(5), 74.

Vaillant, L., \& Samimi, M. (2016). Aphtes et ulcérations buccales. La Presse Médicale, 45 (2), 215-226. doi: 10.1016 / j.lpm.2016.01.005.

Vieira, A. C. F., do Carmo, C. D. S., Vieira, G. M. B., Lima, L. A., da Cruz, M. C. F. N., \& ferreira Lopes, F. (2015). Tratamento da estomatite aftosa recorrente: uma revisão integrativa da literatura. Revista da Faculdade de Odontologia-UPF, 20(3). 